\title{
Proteins: histones and chromatin
}

\section{Editorial overview Patrick Cramer and Cynthia Wolberger}

\author{
Current Opinion in Structural Biology 2011, \\ 21:695-697 \\ Available online 11th November 2011 \\ 0959-440X/\$ - see front matter \\ (C) 2011 Elsevier Ltd. All rights reserved. \\ DOI 10.1016/j.sbi.2011.10.006
}

\section{Patrick Cramer ${ }^{1}$ and Cynthia Wolberger $^{2}$ \\ ${ }^{1}$ Ludwig Maximilians University of Munich,} Gene Center, Department of Biochemistry, Feodor-Lynen-Str. 25, 81377 Munich, Germany

${ }^{2}$ Department of Biophysics and Biophysical Chemistry, Howard Hughes Medical Institute, Johns Hopkins University School of Medicine, 725 N. Wolfe St., Baltimore, MD 21205, USA e-mail: cwolberg@jhmi.edu

Patrick Cramer is a professor of Biochemistry at the Ludwig Maximilians University of Munich, Germany, and head of the Gene Center Munich. He is a structural biologist who has contributed to our understanding of the structure and function of RNAP II from yeast and its associated factors. In a recent study, his laboratory described the structure of RNAP II in complex with the initiation factor TFIIB and proposed a mechanism for the initiation of gene transcription in eukaryotes.

Cynthia Wolberger is a professor of Biophysics and Biophysical Chemistry at the Johns Hopkins University School of Medicine in Baltimore, MD, USA and an investigator in the Howard Hughes Medical Institute. She is a structural biologist who has long studied transcriptional regulation, more recently focusing on enzymes involved in posttranslational modification of histones including deacetylation and deubiquitination. Her laboratory also studies the assembly, disassembly and recognition of different types of polyubiquitin chains.
The packaging of eukaryotic DNA into chromatin presents a formidable barrier to enzymes that must access the DNA template - such as RNA and DNA polymerase - while at the same time providing an opportunity to regulate transcription and DNA replication through the enzymatic modification of chromatin structure. Thanks to tremendous advances over the past two decades in chromatin biology, we now know much about how chromatin activity and the higher-order packaging of nucleosomes is governed by enzymes that assemble the core histone octamer on DNA, reposition nucleosomes, and add or remove post-translational histone modifications. In this issue of Current Opinion in Structural Biology, we focus on the proteins that manipulate chromatin, covering recent advances in understanding the underlying structural and mechanistic basis of chromatin assembly and modification, as well as of the specialized binding domains that recognize particular histone modifications. We have used chromatin organization as a model for organizing the reviews, beginning with discussions of nucleosome remodelling enzymes, which reposition nucleosomes on DNA, followed by reviews on the histone chaperones that assemble nucleosomes. These are followed by reviews on the addition, removal and recognition of dynamic post-translational modification of histones, which play a central role in transcription regulation.

To assemble, disassemble, and reassemble histone octamers and nucleosomes, cells use a variety of histone chaperones. In recent years, it has been increasingly recognized that histone chaperones not only function during the biogenesis of chromatin, but also disassemble nucleosomes during transcription and then reassemble them in the wake of the transcribing RNA polymerase. In their review, Maria Hondele and Andreas Ladurner provide a comprehensive overview of histone chaperones and how they deliver histone monomers or multimers to their appropriate sites while at the same time preventing inappropriate associations with other proteins and nucleic acids. A recurring theme that has emerged from structural studies of chaperones such as Asf1, an $\mathrm{H} 3-\mathrm{H} 4$ chaperone, and Nap1, an H2A-H2B chaperone, is that these assembly proteins directly block the sites on H2A$\mathrm{H} 2 \mathrm{~B}$ dimers, and on $\mathrm{H} 3-\mathrm{H} 4$ dimers and tetramers, that would otherwise bind to DNA or to other histones. A challenge for future studies will be to understand how histone-bound chaperone complexes interact with other proteins, thereby coordinating nucleosome assembly and disassembly with transcription and DNA replication.

Chromatin remodelling complexes use the energy derived from ATP hydrolysis to reposition nucleosomes on DNA. Repositioning a nucleosome requires some form of sliding the histone octamer along the DNA while maintaining the wrapping of the DNA around the histone core. The central 
and still-unanswered question is: by what sequence of chemical and conformational changes, in both the nucleosome and the remodeler, does this transition occur? The large size of chromatin remodelling complexes and the conformational differences among differently liganded states makes addressing mechanistic questions quite challenging, but complementary information from Xray crystallography and electron microscopy has led to testable models of nucleosome remodelling and repositioning. The review by Andres Leschziner covers recent advances in using electron microscopy to study the overall structure and organization of chromatin remodelers. These studies have revealed key features of the overall architecture of these complexes, as well as the potential location of nucleosome binding. Some of the most interesting findings have come from studies of members of the ISWI class of chromatin remodelling enzymes, where conformational differences in the relative position of the ATPase domain appear to depend upon the length of the linker DNA that connects two adjacent nucleosomes. Many questions remain: for example, the combined results from electron microscopy and other biochemical and biophysical studies have given rise to two distinct proposals for the mechanism of nucleosome spacing by the ACF and ISW1a remodelers. Further studies will be needed to resolve whether these two complexes indeed reposition nucleosomes by distinct mechanisms.

Glenn Hauk and Gregory Bowman review recent advances in X-ray crystallographic studies of the ATPase subunits of chromatin remodelers, which have advanced our understanding how these enzymatic domains interact with DNA and are regulated. In their review of structural studies of the SWI2/SNF2 ATPase motors, the authors point to parallels with the structurally related DEAD-box RNA helicases, which provide additional clues to how remodelers may interact with nucleic acid. A recent structure of a fragment of the CHD1 chromatin remodeler that includes both the ATPase domain and a chromodomain, which binds to methylated histone tails, suggests an intriguing autoinhibitory mechanism that may enable CHD1 to discriminate between nucleosomal and naked DNA. As the authors note, we must look to further structures of different SWI/SNF class remodelers in both inhibited and uninhibited states, as well as in complex with DNA and nucleosomal substrates, to establish the specifics of how these enzyme complexes disrupt chromatin.

Histone acetylation and deacetylation are key modifications in regulating transcription and nucleosome assembly. While histone acetyltransferases typically target histones within intact nucleosomes, an interesting exception is the fungal histone acetyltransferase, Rtt109, which must be activated by associating with a histone chaperone. Sheena D'Arcy and Karolin Luger cover recent advances in understanding how binding of the chaperones, Asf1 or Vps75, activates Rtt109. Recent structures of Rtt109 bound to Vps75 suggest that the chaperone activates the acetyltransferase by positioning histone $\mathrm{H} 3$ in the active site, although it is not yet clear whether the same mechanism will hold for Asf1. The central role of histone deacetylases in repressing transcription has made them attractive drug targets for the treatment of cancer, neurodegeneration, and other diseases. The review by Lombardi, Cole, Dowling and Christianson covers recent studies of histone deacetylases and of related bacterial deacetylases, which have provided important new information on active site geometry and catalytic mechanism. Together with structures of deacetylases bound to inhibitors, the most recent findings provide a rich basis for future design of tight-binding inhibitors that may show promise as therapeutic agents.

Histone methylation is both chemically and functionally more complex than acetylation: up to three methyl groups can be attached to a single lysine, and the marks can either repress or activate transcription, depending upon the precise lysine residue that is modified. The review by Sepideh Khorasanizadeh concentrates on recent structural insights into how methylated histone tails are recognized. In particular, it discusses three recent examples of structures that illustrate how lysine-methylated histone tails interact with target proteins. The author observes that the recently described interaction modes are more complex than the canonical interaction of a methylated side chain with an aromatic cage in the target protein. In a concluding note, the author contrasts the multiple structures available for methyl-lysine binding domains with our very limited understanding of how methylated arginine is recognized, a gap that will hopefully soon be narrowed by new structural studies.

The cross-talk between recognition of a histone modification and the addition or removal of another is a core feature of many gene regulatory pathways. As described in the review by Upadhyay, Horton, Zhang and Cheng, many histone demethylases contain additional domains that bind to particular methylated domains, physically coupling 'readers' and 'erasers' in ways that can be exploited to regulate demethylation of the target lysine. The authors cover structural studies that show how specific binding to methylated histone tails, and even to DNA, is exploited to target demethylase enzymes. Another notable development that is highlighted is the more recently discovered role of phosphorylation in regulating the activity of a Jumonji-class demethylase, PHF2, raising the possibility that other demethylases may similarly be regulated by post-translational modifications. The authors speculate that a possible candidate is Tet1, a Jumonji-class enzyme that was unexpectedly found to catalyze the conversion of 5-methylcytosine to 5-hydroxymethylcytosine in DNA. Tet1 contains a 
sequence similar to the C-terminal domain (CTD) of RNA polymerase II, whose function in transcription is regulated by phosphorylation and arginine methylation. It will be interesting to see whether similar modifications regulate the activity or targeting of this fascinating enzyme.

Recent studies also provide insights into the way proteins recognize histone tails bearing post-translational modifications other than the canonical acetylation and methylation marks. In his review, Mark Bycroft describes the structural basis of these interactions, focusing on the recognition of histone tails that are modified by ubiquitination, phosphorylation and poly-ADP ribosylation. In addition to playing a role in transcription regulation, these histone modifications also occur in the DNA damage response and are thought to help recruit repair proteins. In comparison with acetyl-lysine recognition, on which the author also provides an update, our understanding of how these other modifications are recognized within their chromatin context is still quite limited. The author concludes that a major future challenge is to investigate how different domains work in combination to recognize histones bearing multiple modifications, which is considered central to understanding the readout of the 'histone code.'

Transcriptional coactivator complexes can contain a remarkably diverse array of subunits that include multiple histone-modifying enzymes and domains that recognize different histone modifications. Structures of individual subunits have been highly informative, but have left open the question of how organization into a larger complex governs function. The final review by Nadine Samara and Cynthia Wolberger describes structural studies of the SAGA coactivator complex, which both acetylates histones and removes monoubiquitin from histone $\mathrm{H} 2 \mathrm{~B}$. The structure of the four-protein subcomplex that deubiquitinates histone $\mathrm{H} 2 \mathrm{~B}$, termed the SAGA DUB module, revealed an unexpected intertwined architecture, which may well prove to be a common theme in further structural studies of SAGA and of other coactivator complexes.

It is exciting to see how far the field has come in establishing the mechanistic basis for chromatin modification, and how changes in chromatin are connected to altered patterns of transcription. Converging approaches of X-ray crystallography and electron microscopy, coupled with a wealth of biochemical data, will make it possible to assemble snapshots from atomic resolution structures in space and in time to reveal how so many complex biochemical events are coordinated to modify chromatin. There are challenges and opportunities aplenty, which ensures that this area of investigation will remain an exciting one for many years to come. 